\title{
Thyroid cytology reporting and diagnostic accuracy: a departmental audit of the rcpath guidelines
}

\begin{abstract}
In the past there has been considerable variation in the way thyroid cytology was reported by pathologists. As the need for improving patient care increased to include more conservative surgical procedures for the management of thyroid lesions, there was a need for a standardized reporting format. This had to be reproducible and universal so that the implications of the reporting categories were clear to all pathologists, surgeons or clinicians. In 2009, The Royal College of Pathologists (RCPath) published 'Guidance on Reporting of Thyroid Cytology Specimens' and this guidance has been revised in 2016.1 This document details the standardized format for reporting Thyroid FNAC used in the UK today. The classification of thyroid cytology into 'Thy categories' is similar to the Bethesda system used in the USA in that it stratifies thyroid FNA's into various disease categories. The UK system uses five main categories ranging from Thy 1-Thy5 and certain categories are further subdivided into $\mathrm{a}$ or $\mathrm{b}$ to give additional diagnostic information. The Bethesda system uses six categories each with a percentage risk of malignancy. Currently there is little national data available on the percentage of cases that fall into each Thy category. Hence the positive predictive value (PPV) for each Thy category suggested in the RCPath guidance although rudimentary is present target.

This audit was conducted at Wycombe Hospital Cellular pathology department to assess the adherence to RCPath 'Guidance on reporting of Thyroid Cytology Specimens'. In addition to the aspects of the audit outlined in the guidance, we have also gathered information on the accuracy of local diagnostic opinions compared to those of specialists at the regional MDT to determine if there are large discrepancies between local and specialist practice.
\end{abstract}

Keywords: neoplastic, papillary carcinoma, diagnostic, audit, cases

\author{
Volume 5 Issue 4 - 2017
}

Gayathri Wathug, Kalani Kuruppu
Buckinghamshire Healthcare NHS Trust, UK

Correspondence: Kalani Kuruppu, Buckinghamshire Healthcare NHS Trust, UK, Email dona_kalani@hotmail.com

Received: June 23, 2017 | Published: December 22, 2017
Abbreviations: RCPath, royal college of pathologists; PPV, positive predictive value; Thy, thyroid cytology reports

\section{Aims or objectives}

i. To determine in what proportion of thyroid cytology reports a "Thy" category is included as well as a prose explanation of the findings.

ii. To determine the percentage of cases that fall into each Thy category.

iii. To correlate cytology with subsequent histology in order to determine diagnostic accuracy and the PPV for neoplasia and malignancy in each Thy category. In addition to the audit criteria, the sensitivity, the specificity, false negative rate, false positive rate and overall accuracy should be calculated and compared to those stated in the RCPath guidance.

iv. To assess the number of Thy3a-Thy 5 cases referred to the regional Thyroid Cancer MDT.

v. To compare the opinion of local pathologists with those of the regional MDT.

\section{Audit criteria}

The agreed criteria range:

i. $100 \%$

ii. To be determined nationally as data becomes available

iii. To be determined nationally as data becomes available. The PPV of Thy 5 for malignancy should, however, be $100 \%$.

Additional parameters ${ }^{2}$

i. Sensitivity for malignancy between $65-98 \%$

ii. Specificity for malignancy between $76-100 \%$

iii. False negative rate between $0-5 \%$

iv. False positive rate between $0-5.7 \%$

v. Overall accuracy of $69-97 \%$

vi. As per local policy, $100 \%$ for all Thy3a, Thy3f, Thy 4 and Thy5cases.

vii. No set standard, however there should not be major discrepancies between local and specialist opinions (Table 1). 
Table I The agreed criteria range

\begin{tabular}{lll}
\hline RCpath category & \% Use of each category & PPV for malignancy \\
\hline Thy $1 / 1 \mathrm{c}$ & $18-22$ & 4 \\
Thy2/2c & $42-51$ & 1.4 \\
Thy3a & 10-May & 17 \\
Thy3f & 14-16 & Up to 40 \\
Thy4 & 4-Feb & Up to 68 \\
Thy5 & 10-May & Up to 100 \\
\hline
\end{tabular}

\section{Methods}

\section{Sample selection}

A search for all thyroid FNAs over a set time period (12/05/14$12 / 05 / 12$ ) was conducted on the local software system (Winpath) using the following search criteria: Series: N, Time Period: 12/05/1412/05/12, T Code: T96000 - Thyroid Gland

Cases where multiple aspirates were received under one lab number were treated as separate cases (i.e. aspirate of left/right sided lesion). The following exclusion criteria were then applied:

i. Cases from April and May 2014 as no subsequent histology was available (shortening the audited time period to $12 / 05 / 12$ 27/03/14).

ii. Cases which were erroneously coded as Thyroid FNA's (Lymph node/salivary gland aspirates).

This yielded a total of 812 cases for review including those which did not have an assigned Thy category. After the results were produced for criteria no. 1, these cases were excluded from the remainder of the audit as criteria 2-4 require a Thy category for analysis.

\section{Data collection}

An audit preformed was adapted from the template provided by the RCPath: 'Audit of reporting of thyroid cytology specimens and their correlation with thyroid histology' to record the following data:

i. Was there a prose report included with the Thy category?

ii. Was there subsequent histology?

iii. If there was subsequent histology, which disease category did the specimen fall into? (non neoplastic, benign neoplasm, papillary thyroid cancer, follicular carcinoma, medullar carcinoma or other)

iv. Was the cytology referred to MDT?

v. If referred to MDT, what was the specialist opinion?

Using the RCPath audit template, the subsequent histology was categorised as follows:

i. Non neoplastic: colloid nodule, hyperplasic nodule, Hashimoto's thyroiditis, multinodular goitre, normal thyroid tissue.

ii. Benign neoplasm: follicular adenoma. iii. Malignant neoplasm: papillary carcinoma, follicular carcinoma, medullar carcinoma, other.

Each case was reviewed from Winpath and the authorised report used to record the relevant data. Subsequent results for the same patient were also checked to enable correlation with histology. If a histology result was present for the same lesion (laterality and site), immediately following the aspirate this was recorded. If however, the patient was investigated with further FNA's; this was recorded as 'no subsequent histology.' Each report was then checked for referral to the regional multidisciplinary team (MDT) meeting (as stated in each report) and the subsequent specialist opinion. Only the cases with a supplementary report including the specialist opinion were regarded as referred to MDT. Those cases where histology was reviewed in hindsight due to discrepancy with the initial cytology were not included as a true referral to MDT.

\section{Statistical calculations}

In order to calculate the required parameters, Thy $1 / 1 \mathrm{c}$ cases were disregarded to avoid providing false reassurance. Thy $2 / 2 \mathrm{c}$ cases were deemed a negative result for malignancy and Thy 3a-Thy 5 cases were considered positive. The diagnosis of a follicular adenoma on subsequent histology was considered a positive result. Thus the following definitions were applied:

a) True Negative (FN): Thy 2/2c cytology cases with subsequent 'non neoplastic' histology results.

b) False Negative (FN): Thy $2 / 2 \mathrm{c}$ cytology cases with 'benign i.e follicular adenoma' or 'malignant' histology.

c) True Positive (TP): Thy 3a-Thy5 cytology cases with subsequent 'benign i.e follicular adenoma' or 'malignant' histology.

d) False Positive (FP): Thy 3a-Thy5 cytology cases with subsequent 'non neoplastic' histology results.

Once the data was divided into these four categories, the following standard calculations were used:

e) Sensitivity: $\mathrm{TP} /(\mathrm{TP}+\mathrm{FN})$

f) Specificity: $\mathrm{TN} /(\mathrm{TN}+\mathrm{FP})$

g) Accuracy: $(\mathrm{TP}+\mathrm{TN}) /(\mathrm{TP}+\mathrm{TN}+\mathrm{FP}+\mathrm{FN})$

h) Positive Predictive Value (PPV): TP/(TP+FP) 


\section{i) False Negative Rate: $\mathrm{FN} /(\mathrm{TN}+\mathrm{FN})$}

j) False Positive Rate: FP/(TP+FP)

\section{Results and discussion}

\section{Adequacy of report}

The majority of cases reviewed (97.7\%) were of adequate quality and included both the Thy category and an accompanying prose report as recommended by the RCPath. The remainder of cases did not include the Thy category however a written report was issued from which the diagnosis could be ascertained. These cases were subsequently removed from the sample as the other audit criteria assess the designated Thy category (Table 2).

\section{Distribution of cases into each thy category}

The distribution of cases is illustrated by Figures 2. The vast majority of Thyroid FNA's received by the Wycombe Pathology department fall into the Thy 2 category (409 cases, 51.58\%). The second largest category is Thy 1 (215 ases, 27.11\%), indicating a significant cost in consultant and lab processing time for samples which are insufficient for diagnostic purposes. Very few cases fall into the Thy 5 category (10cases, $1.26 \%$ ) and even fewer are categorized as Thy 4 ( 5 cases, $0.63 \%)$. Thy 3 lesions are predominantly designated Thy $3 \mathrm{f}$ (50cases, $6.31 \%$ ) with the provision that a follicular lesion cannot be excluded.

This is comparable to a similar audit carried out at NHS Tayside. ${ }^{3}$ The author of this Scottish study found the largest proportion of their specimens were Thy 2 (44\%), followed by Thy3 (26\%), Thy 1 (25\%), Thy4 and Thy5 (2.5\% each) (Table 3$)$.

In comparison to the percentage usage of each category by the RCPath, our data range extends beyond theirs for Thy 1-2c categories and is lower for than the RCPath for Thy3a-5cases. The reasons for this may be multifactorial including the skill level of those taking the FNA at different trusts, the availability of expert cytopathologists at tertiary centres and varying degrees of confidence in classifying indeterminate cases.

\section{Correlation with histology and PPV}

Unfortunately, a significant proportion of the sample did not have subsequent histology (650cases). This is correlates with the substantial Thy1 (276cases) and Thy2 (239cases)... which would not be managed by surgical excision. Excluding Thy $1 / 1 \mathrm{c}$ cases, in those which had subsequent histology, 51 were non neoplastic lesions and 35 cases were benign follicular adenomas. 13cases proved to be papillary carcinomas and 5 were follicular carcinomas. Within the Thy 5 category (10cases), 3 did not have subsequent histology and the remaining 7 cases were all malignant (6 papillary carcinomas, 1 other) which establishes $100 \%$ diagnostic accuracy in these cases, as is the set standard.

For the Thy3f category, none of the cases were subsequently diagnosed as follicular carcinomas. There were however four Papillary carcinomas and 1 'other' malignancy. The vast majority of these (22cases) were benign follicular adenomas. The 'other' malignancies encountered included a lymphoma, anaplastic carcinoma and metastasis from a primary elsewhere. The results for each Thy category and the subsequent histology are shown in Figure 3, (Table 4).
In this audit, the FNA was viewed as a screening tool for determining which thyroid lesions required surgical excision. Thereby, only Thy2/2c FNA's were considered 'negative'. The PPV for both neoplastic lesions (i.e including follicular adenomas and malignancies) and for malignant lesions alone was calculated for Thy $3 \mathrm{a}-5$ categories. A PPV for Thy $1-2 \mathrm{c}$ is not provided as the authors were unable to find Table 5 evidence of how these were calculated in RCPath guidelines or the definitions used to determine them.

When compared to a large multicentre study done in $2010,{ }^{4}$ which found that the risk of malignancy for 'indeterminate' cytology cases was $34 \%$ and $98 \%$ in malignant cytology cases our PPV for neoplasia in Thy 3 cases of $75 \%$ is much higher . Our PPV for malignancy in Thy3a cases is also much higher than the RCPath standards. The PPV for Thy 4 was lower at $50 \%$ however this is likely to be secondary to the small number of cases within this category. We were compliant with the RCPath standard for Thy5 of having a 100\% PPV for this category (Table 5).

When the additional parameters were calculated we achieved the targets set by the RCPath guidance for specificity and overall accuracy only. The other parameters fell outside the RCPath targets. The overall sensitivity of $64 \%$ fell short of the target range. The false negative rate of $31 \%$ was much higher than the target of $0-5 \%$ and the false positive rate was higher than recommended at $23 \%$. Compared to a similar study in 2008 which found a sensitivity of $92.6 \%$, a specificity of $91.6 \%$ and a false negative rate of $2.3 \%$, our local department rates need significant improvement, particularly for sensitivity and false positive/negative rates. A specificity of $80 \%$ suggests that local pathologists are good at identifying true negative cases. Although the sensitivity rate is lower than the target range, it is only so by $1 \%$, suggesting that positive cases are in fact being picked up well and a larger sample size might yield higher results. The high false negative/positive rates may be explained by the inclusion of benign follicular adenomas as positive results especially as $31 \%$ of all follicular adenomas in our sample came from a Thy2 (negative) FNA. The overall accuracy of $72 \%$ falls within the required range and thus the department is performing adequately, but results could be markedly better. These results are limited by the small number of cases used (143cases) to calculate these statistics as all those without histology (650cases) were not included. It is also important to note that the target ranges provided by the RCPath guidance is likely to evolve as more data becomes available in the future (Table 6).

\section{Referral to regional MDT}

The standards set by the RCPath suggest that $100 \%$ of Thy 4 and Thy 5 cases should be referred to MDT. As per local protocols, Thy3a and Thy $3 \mathrm{f}$ should also be referred. The results show that $92 \%$ of Thy 3 a, $90 \%$ of Thy 3 f, $80 \%$ of Thy 4 and $100 \%$ of Thy 5 cases were referred to MDT and a subsequent specialist opinion was available (Figure 1) \& (Figure 2).

\section{Correlation with specialist opinion}

In addition to the set standards, we compared the initial diagnostic opinion of the local pathologists with the final diagnostic opinion of the specialists at the regional MDT to determine if there were major discrepancies. When cases were initially given a Thy 5 , there was agreement with the specialists for $90 \%$ of these cases. Only one case was downgraded to Thy4. Of the four Thy 4 cases which were sent to MDT, two cases were agreed as Thy4, one case was upgraded to 
Thy5 and one case was downgraded to Thy3a. This result is difficult to interpret accurately as there are so few numbers of cases in this category. However, downgrading to Thy 3 a will confer a difference in management for the patient who may subsequently undergo a hemithyroidectomy instead of a total thyroidectomy. ${ }^{5-7}$

After 12 Thy3a cases were submitted for specialist opinion, three were upgraded and two were downgraded. This means a $41.6 \%$

Table 2 Table showing the quality of reports compared to audit standards diagnostic resolution of these indeterminate cases. There were a total of 45 Thy $3 \mathrm{f}$ cases referred to MDT. 57\% were agreed as Thy $3 \mathrm{f}$, $27 \%$ were downgraded and $16 \%$ were upgraded. This infers a $43 \%$ diagnostic resolution. These statistics compare with a published literature review which found a $42.5 \%$ diagnostic resolution on 'indeterminate' cases sent for second opinion. It was outside the scope of this audit to compare the subsequent histology in cases where there was discrepancy between local and regional opinion (Figure 3).

\begin{tabular}{llll}
\hline Quality of report & No. of cases & Percentage of total (\%) & Audit standard (\%) \\
\hline Prose Report and Thy Category & 793 & 97.7 & 1000 \\
Prose Report Only & 19 & 2.3 & 0 \\
Thy Category Only & 0 & 0 & 0
\end{tabular}

Table 3 Table showing proportion of cases within each Thy category

\begin{tabular}{llll}
\hline Thy category & No. of cases & \% Use of category & RCpath \% usage \\
\hline 1 & 215 & 27.11 & $18-22$ \\
$1 \mathrm{c}$ & 61 & 7.69 & \\
2 & 409 & 51.58 & $42-51$ \\
$2 \mathrm{c}$ & 30 & 3.78 & $5-10$ \\
$3 \mathrm{a}$ & 13 & 1.64 & $14-16$ \\
$3 \mathrm{f}$ & 50 & 6.31 & $2-4$ \\
4 & 5 & 0.63 & $5-10$ \\
\hline
\end{tabular}

Table 4 Table of correlation between histology and cytology

\begin{tabular}{llllllllll}
\hline Histology & Thy $\mathbf{1}$ & Thy $\mathbf{1 c}$ & Thy $\mathbf{2}$ & Thy 2c & Thy 3a & Thy 3f & Thy 4 & Thy $\mathbf{5}$ & Total \\
\hline No Histology & 188 & 52 & 353 & 27 & 9 & 15 & 3 & 3 & 650 \\
Non Neoplastic & 18 & 7 & 38 & 3 & 1 & 8 & 1 & 0 & 76 \\
Benign & 8 & 1 & 11 & 0 & 2 & 22 & 0 & 0 & 44 \\
Ptc & 1 & 1 & 2 & 0 & 1 & 4 & 0 & 6 & 15 \\
Fc & 0 & 0 & 5 & 0 & 0 & 0 & 0 & 0 & 5 \\
MTC & 0 & 0 & 0 & 0 & 0 & 0 & 0 & 0 & 0 \\
Other & 0 & 0 & 0 & 0 & 0 & 1 & 1 & 1 & 3 \\
Total & 215 & 61 & 409 & 30 & 13 & 50 & 5 & 10 & 793 \\
\hline
\end{tabular}


Table 5 Table of positive predictive values

\begin{tabular}{lllll}
\hline Category & $\begin{array}{l}\text { PPV for neoplasia } \\
(\%)\end{array}$ & $\begin{array}{l}\text { PPV for malignancy } \\
(\%)\end{array}$ & $\begin{array}{l}\text { RCPath PPV for malignancy } \\
(\%)\end{array}$ & $\begin{array}{l}\text { Bethesda - associated risk of } \\
\text { malignancy }\end{array}$ \\
\hline Thy3a & 75 & 25 & 17 & $5-15$ \\
Thy3f & 77 & 14.3 & Up to 40 & $15-30$ \\
Thy & 50 & 50 & Up to 68 & $60-75$ \\
Thy5 & 100 & 100 & Up to 100 & $97-100$ \\
\hline
\end{tabular}

Table 6 Table of additional parameters compared to target range

\begin{tabular}{lll}
\hline Parameters & Percentage (\%) & RCPath targets (\%) \\
\hline Sensitivity & 64 & $65-98$ \\
Specificity & 80 & $76-100$ \\
False Negative Rate & 31 & $0-5$ \\
False Positive Rate & 23 & $0-5.7$ \\
Overall Accuracy & 72 & $69-97$ \\
\hline
\end{tabular}

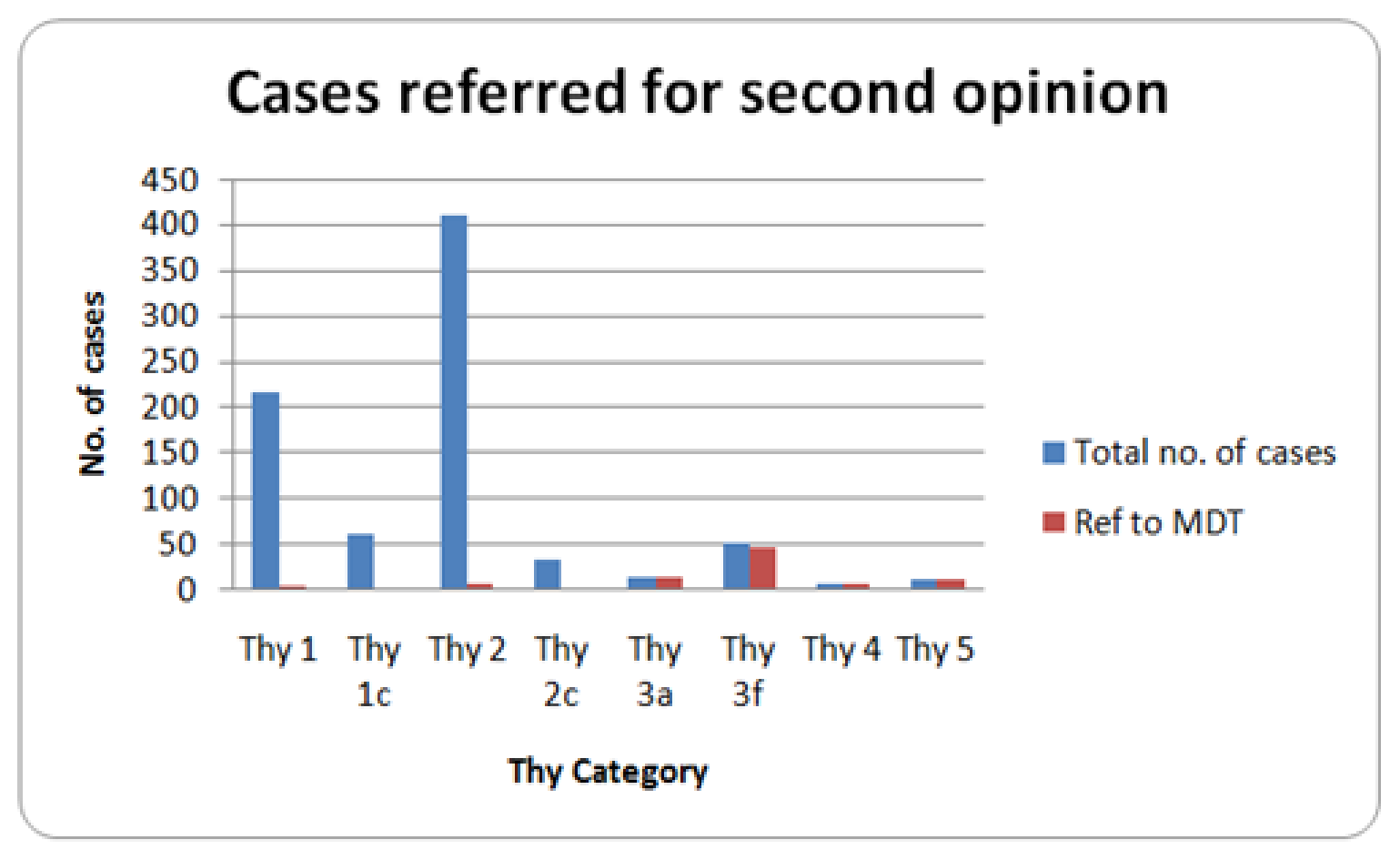

Figure I Graph of all cases referred to MDT. 


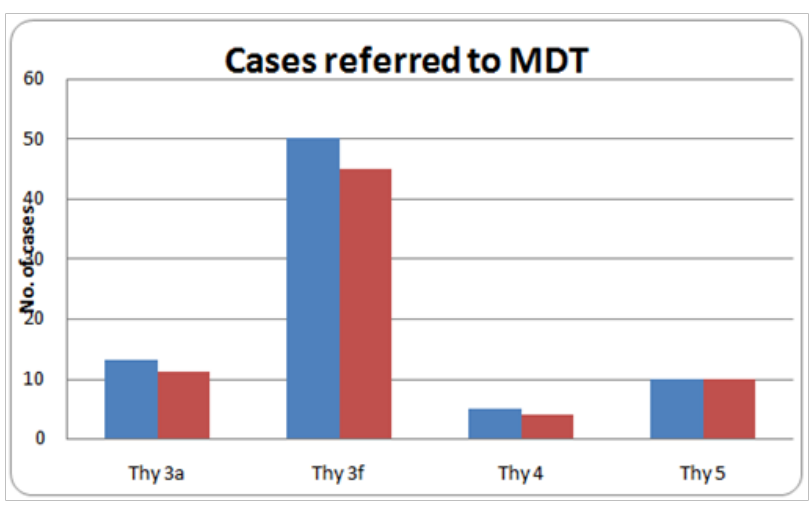

Figure 2 Graph of all cases which should be referred to MDT.

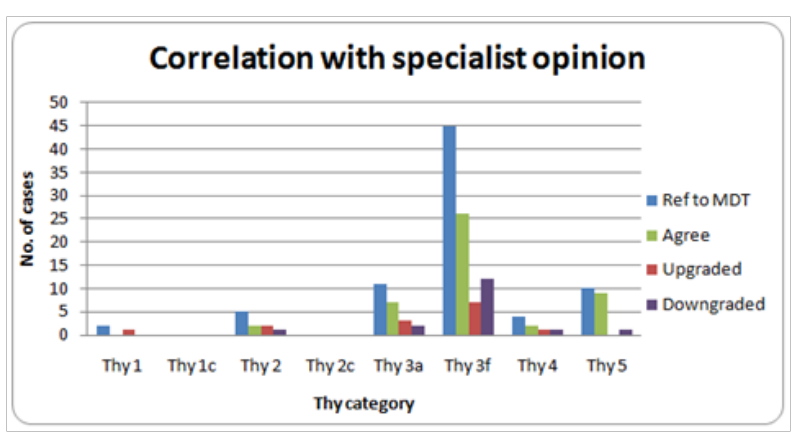

Figure 3 Graph showing the variability between local and regional opinions of those cases referred to MDT.

\section{Conclusion}

This audit shows that the department is $97.7 \%$ compliant with audit criterion 1 which requires a prose report to accompany the Thy category. The percentage usage of each category is vastly different at our trust to those stated by the RCPath. PPVs for malignancy in our sample were either higher than or compliant with the RCPath standards. In addition, we have achieved the targets for specificity $(80 \%)$ and overall accuracy (72\%) however the sensitivity $(64 \%)$, the false negative rate $(31 \%)$ and the false positive rate $(23 \%)$ fall far outside the recommended range. We achieved $100 \%$ compliance with Criterion 4, but only for Thy 5 cases. All other categories (Thy 3a, Thy3f and Thy 4) included some cases which should have been referred to MDT but for unknown reasons were not sent. The value of referral to regional experts was highlighted by a $41.6 \%$ and $43 \%$ diagnostic resolution of Thy $3 \mathrm{a}$ and Thy $3 \mathrm{f}$ cases respectively.

\section{Recommendations}

i. Continue to collect data on the proportion of cases which fall into each Thy category to be contributed to national data.

ii. To improve the PPV of Thy3a-Thy4 cases, consider encouraging attendance at regional MDT's, teaching sessions and courses to train staff to increase diagnostic accuracy.

iii. Inform all medical staff and administrative staff which cases should be referred to MDT. Develop a Performa which would trigger an automatic referral to MDT for the relevant cases.

iv. Re-audit in $12-18$ months.

\section{Acknowledgements}

None.

\section{Conflict of interest}

The author declares they have no actual or potential competing interests.

\section{References}

1. Cross P, Chandra A, Giles T, et al. Guidance on the reporting of thyroid cytology specimens. England: Royal College of Pathologists; 2016.

2. Royal college of pathologists. Guidance on the reporting of thyroid cytology specimens. USA; 2009.

3. Allan R. The accuracy of fine needle aspiration at identifying thyroid malignancy in Tayside. Scottish Universities Medical Journal. 2012;1(1):104-113.

4. Syed Z Ali, Edmund S Cibas. The Bethesda System for Reporting Thyroid Cytopathology. Springer; 2010.

5. Wang CC, Friedman L, Kennedy GC. A large multicentre correlation study of thyroid nodule Cytopathology and histopathology. Thyroid. 2011;21(3):243-251.

6. Haberal AN, Toru S, Ozen O, et al. Diagnostic pitfalls in the evaluation of fine needle aspiration cytology of the thyroid: correlation with histopathology in 260 cases. Cytopathology. 2009;20(2):103-108.

7. Gerhard, Rene, Boerner, Scott $\mathrm{L}$. The value of second opinion in Thyroid cytology: a review. Cancer Cytopathology. 2014;122(8):611-619. 